\title{
CERTAIN SUBCLASSES OF MULTIVALENT FUNCTIONS INVOLVING LINEAR OPERATORS
}

\author{
H. A. AL-KHARSANI AND N. M. AL-AREEFI
}

\begin{abstract}
In terms of linear operators we introduce new classes of functions. Then by using differential subordinations, certain results concerning inclusion relations, coefficient bounds and other results are given.
\end{abstract}

\section{Introduction}

Let $\mathcal{A}_{p}$ denote the classes of functions of the form

$$
f(z)=z^{p}+\sum_{k=p+1}^{\infty} a_{k} z^{k}
$$

which are analytic in the open disk $\mathbb{U}=\{z \in \mathbb{C},|z|<1\}$. If $f$ and $g$ are analytic in $\mathbb{U}$, we say that $f$ is subordinate to $g$, written $f \prec g$ or $f(z) \prec g(z)$, if there exists a Schawrz function $w$, analytic in $\mathbb{U}$ which $w(0)=0$ and $|w(z)|<1(z \in \mathbb{U})$, such that $f(z)=g(w(z))(z \in \mathbb{U})$. In particular, if the function $g$ is univalent in $\mathbb{U}$, the above subordination is equivalent to $f(0)=g(0)$ and $f(\mathbb{U}) \subset g(\mathbb{U})$. For $0 \leq \xi<p, \beta<1$, we denote by $\chi^{*}(\xi), \mathcal{K}(\xi), \Phi(\xi, \beta)$ the subclasses of $\mathcal{A}_{p}$ consisting of all analytic $p$-valent functions which are, respectively, starlike of order $\xi$, convex of order $\xi$, close-to-convex of order $\xi$, and type $\beta$ in $\mathbb{U}$.

Let $\mathcal{N}$ be the class of all functions $\phi$ which are analytic and univalent in $\mathbb{U}$ and for which $\phi(\mathbb{U})$ is convex with $\phi(0)=1$ and $\operatorname{Re}\{\phi(z)\}>0$ for $z \in \mathbb{U}$.

From the principle of subordination between analytic functions, we introduce the subclasses $\chi^{*}(\xi, \phi), \mathcal{K}(\xi, \phi)$, and $\phi(\xi, \beta, \phi, \psi)$ of the class $\mathcal{A}_{p}$ for $0 \leq \xi<p, \beta<1$, and

Corresponding author: N. M. Al-Areefi.

Received March 11, 2009; revised November 25, 2009.

2000 Mathematics Subject Classification. 30C80, 30C45.

Key words and phrases. Analytic function, $p$-valent function, differential subordinates, differential superordinations, Dziok-Srivastava linear operator, multiplier transformation. 
$\phi, \psi \in \mathcal{N}$, which are defined by

$$
\begin{aligned}
\chi^{*}(\xi ; \phi) & :=\left\{f \in \mathcal{A}_{p}: \frac{1}{p-\xi}\left(\frac{z f^{\prime}(z)}{f(z)}-\xi\right) \prec \phi(z) \text { in } \mathbb{U}\right\}, \\
\mathcal{K}(\xi ; \phi) & :=\left\{f \in \mathcal{A}_{p}: \frac{1}{p-\xi}\left(1+\frac{z f^{\prime \prime}(z)}{f^{\prime}(z)}-\xi\right) \prec \phi(z) \text { in } \mathbb{U}\right\}, \\
\Phi(\xi, \beta ; \phi, \psi) & :=\left\{f \in \mathcal{A}_{p}: \exists g \in \chi^{*}(\xi, \phi) \text { s.t. } \frac{1}{p-\beta}\left(\frac{z f^{\prime}(z)}{g(z)}-\beta\right) \prec \psi(z) \text { in } \mathbb{U}\right\} .
\end{aligned}
$$

We note that the classes mentioned above are the familiar classes which have been used widely on the space of analytic and $p$-valent functions in $\mathbb{U}$, and for special choices for the functions $\phi$ and $\psi$ involved in these definitions, we can obtain the well known subclasses of $\mathcal{A}_{p}$. For example, we have

$$
\chi^{*}\left(\xi ; \frac{1+z}{1-z}\right)=\chi^{*}(\xi), \mathcal{K}\left(\xi ; \frac{1+z}{1-z}\right)=\mathcal{K}(\xi), \Phi\left(\xi, \beta ; \frac{1+z}{1-z}, \frac{1+z}{1-z}\right)=\Phi(\xi, \beta) .
$$

Also let the Hadamard product (or convolution) $f * g$ of two analytic functions

$$
f(z)=z^{p}+\sum_{k=p+1}^{\infty} a_{k} z^{k}, g(z)=z^{p}+\sum_{k=p+1}^{\infty} b_{k} z^{k}
$$

be given by

$$
(f * g)(z)=z^{p}+\sum_{k=p+1}^{\infty} a_{k} b_{k} z^{k}
$$

For $\alpha_{j} \in \mathbb{C}(j=1,2, \ldots, l)$ and $\beta_{j} \in \mathbb{C} \backslash\{0,-1,-2, \ldots\}(j=1,2, \ldots, m)$, the generalized hypergeometric function is defined by the infinite series

${ }_{l} F_{m}\left(\alpha_{1}, \ldots, \alpha_{l} ; \beta_{1}, \ldots, \beta_{m} ; z\right)=\sum_{k=0}^{\infty} \frac{\left(\alpha_{1}\right)_{k} \cdots\left(\alpha_{l}\right)_{k}}{\left(\beta_{1}\right)_{k} \cdots\left(\beta_{m}\right)_{k}} \frac{z^{k}}{k !}\left(l \leq m+1 ; l, m \in \mathbb{N}_{0}=\{0,1,2, \ldots\}\right)$,

where $(a)_{k}$ is the Pochhammer symbol defined by

$$
(a)_{k}:=\frac{\Gamma(a+k)}{\Gamma(k)} \begin{cases}1 & (k=0) \\ a(a+1) \cdots(a+k-1) & (k \in \mathbb{N}:=\{1,2, \ldots\} .\end{cases}
$$

In particular, the incomplete beta function, related to the Gauss hypergeometric function, $\phi(a, c ; z)$ is defined by

$$
\phi(a, c ; z)=z F(a, 1 ; c ; z)=\sum_{k=0}^{\infty} \frac{(a)_{k}}{(c)_{k}} z^{k+1}, z \in \mathbb{U}, c \neq 0,-1,-2, \ldots
$$

Note that $\phi(a, 1 ; z)=\frac{z}{(1-z)^{a}}$. Moreover, $\phi(2,1 ; z)=\frac{z}{(1-z)^{2}}$ is the Koebe function. 
Corresponding to the function

$$
h_{p}\left(\alpha_{1}, \ldots, \alpha_{l} ; \beta_{1}, \ldots, \beta_{m} ; z\right):=z^{p}{ }_{l} F_{m}\left(\alpha_{1}, \ldots, \alpha_{l} ; \beta_{1}, \ldots, \beta_{m} ; z\right) .
$$

The Dziok-Srivastava operator [7] (see also [6] and [22]) $H_{p}^{l, m}\left(\alpha_{1}, \ldots, \alpha_{l}, \beta_{1}, \ldots, \beta_{m}\right.$ ) is defined by the Hadamard product

$$
\begin{aligned}
H_{p}^{l, m}\left(\alpha_{1}, \ldots, \alpha_{l} ; \beta_{1}, \ldots, \beta_{m}\right) f(z) & \\
& =h_{p}\left(\alpha_{1}, \ldots, \alpha_{l} ; \beta_{1}, \ldots, \beta_{m} ; z\right) * f(z) \\
& =z^{p}+\sum_{k=p+1}^{\infty} \frac{\left(\alpha_{1}\right)_{k-p} \cdots\left(\alpha_{l}\right)_{k-p}}{\left(\beta_{1}\right)_{k-p} \cdots\left(\beta_{m}\right)_{k-p}} \frac{a_{m} z^{k}}{(k-p) !} .
\end{aligned}
$$

Special cases of the Dziok-Srivastava linear operator include the Hohlov linear operator [9]. The Carlson-Shaffer linear operator [3], the Ruscheweh derivative operator [19], the generalized Bernardi-Libera-Livingston operator (cf. [2], [11], [12]) and the Srivastava-Owa fractional derivative operators (cf. [16], [18]).

Corresponding to the function $h_{p}\left(\alpha_{1}, \ldots, \alpha_{l}, \beta_{1}, \ldots, \beta_{m} ; z\right)$ defined by (1.6), we introduce a function $\mathcal{F}_{\mu}\left(\alpha_{1}, \ldots, \alpha_{l}, \beta_{1}, \ldots, \beta_{m} ; z\right)$ given by

$$
\begin{gathered}
h_{p}\left(\alpha_{1}, \ldots, \alpha_{l} ; \beta_{1}, \ldots, \beta_{m} ; z\right) * \mathcal{F}_{\mu}\left(\alpha_{1}, \ldots, \alpha_{l} ; \beta_{1}, \ldots, \beta_{m} ; z\right) \\
=\frac{z^{p}}{(1-z)^{\mu+p-1}},(\quad z \in \mathbb{U}, \mu>0, l=m+1) .
\end{gathered}
$$

Analogous to $H_{p}\left(\alpha_{1}, \ldots, \alpha_{l}, \beta_{1}, \ldots, \beta_{m}\right)$, we now define the linear operator $J_{\mu}\left(\alpha_{1}, \ldots ; \alpha_{l} ; \beta_{1}, \ldots, \beta_{m}\right)$ on $\mathcal{A}_{p}$ as follows:

$$
\begin{aligned}
& J_{\mu}\left(\alpha_{1}, \ldots, \alpha_{l}, \beta_{1}, \ldots, \beta_{m}\right) f(z)=\mathcal{F}_{\mu}\left(\alpha_{1}, \ldots, \alpha_{l}, \beta_{1}, \ldots, \beta_{m} ; z\right) * f(z) \\
& \left(\alpha_{1}, \beta_{j} \in \mathbb{C} \backslash \mathbb{Z}_{0}^{-} ; i=1, \ldots, l ; j=1, \ldots, m ; \mu>0 ; z \in \mathbb{U} ; f \in \mathcal{A}_{p}\right) .
\end{aligned}
$$

For convenience, we write

$$
J_{\mu}^{l, m}\left(\alpha_{1}\right):=J_{\mu}\left(\alpha_{1}, \ldots, \alpha_{l}, \beta_{1}, \ldots, \beta_{m}\right) .
$$

If $f$ is given by (1.1), then by (1.8), we see that

$$
J_{\mu}^{l, m}\left(\alpha_{1}\right) f(z)=z^{p}+\sum_{k=p+1}^{\infty} \Psi_{k}\left(\alpha_{1}, \mu\right) a_{k} z^{k},
$$

where

$$
\Psi_{k}\left(\alpha_{1}, \mu\right)=(\mu+p-1)_{k-p} \frac{\left(\beta_{1}\right)_{k-p} \cdots\left(\beta_{m}\right)_{k-p}}{\left(\alpha_{1}\right)_{k-p} \cdots\left(\alpha_{l}\right)_{k-p}} .
$$

Special cases of this operator are [10] when $p=1$, the generalized integral operator in [1] when $p=1$ and $\mu=2$ and Noor integral operator [14]. We can verify from the definition (1.8) that

$$
\begin{aligned}
z\left(J_{\mu}^{l, m}\left(\alpha_{1}+1\right) f(z)\right)^{\prime} & =\alpha_{1} J_{\mu}^{l, m}\left(\alpha_{1}\right) f(z)-\left(\alpha_{1}-p\right) J_{\mu}^{l, m}\left(\alpha_{1}+1\right) f(z) \\
z\left(J_{\mu}^{l, m}\left(\alpha_{1}\right) f(z)\right)^{\prime} & =(\mu+p-1) J_{\mu+1}^{l, m}\left(\alpha_{1}\right) f(z)-(\mu-1) J_{\mu}^{l, m}\left(\alpha_{1}\right) f(z)
\end{aligned}
$$


By using the operator $J_{\mu}^{l, m}\left(\alpha_{1}\right)$, we introduce the following classes of analytic functions for $\phi, \psi \in \mathcal{N}$ and $0 \leq \xi<p, \beta<1$ :

$$
\begin{aligned}
\chi_{\alpha_{1}, \mu}(l, m ; \xi ; \phi) & :=\left\{f \in \mathcal{A}_{p}: J_{\mu}^{l, m}\left(\alpha_{1}\right) f(z) \in \chi^{*}(\xi ; \phi)\right\} \\
\mathcal{K}_{\alpha_{1}, \mu}(l, m ; \xi ; \phi) & :=\left\{f \in \mathcal{A}_{p}: J_{\mu}^{l, m}\left(\alpha_{1}\right) f(z) \in \mathcal{K}(\xi ; \phi)\right\} \\
\Phi_{\alpha_{1}, \mu}(l, m ; \xi, \beta ; \phi, \psi) & :=\left\{f \in \mathcal{A}_{p}: J_{\mu}^{l, m}\left(\alpha_{1}\right) f(z) \in \Phi(\xi, \beta ; \phi, \psi)\right\} .
\end{aligned}
$$

Also

$$
f(z) \in \mathcal{K}_{\alpha_{1}, \mu}(l, m ; \xi ; \phi) \Leftrightarrow \frac{z f^{\prime}(z)}{p} \in \chi_{\alpha_{1}, \mu}(l, m ; \xi ; \phi) .
$$

The multiplier transformations defined on $\mathcal{A}_{p}$ by the following infinite series

$$
I_{p}(r, \lambda) f(z)=z^{p}+\sum_{k=p+1}^{\infty}\left(\frac{k+\lambda}{p+\lambda}\right)^{r} a_{k} z^{k} \quad(\lambda \geq 0) .
$$

The operator $I_{p}(r, \lambda)$ is closely related to Şălăgean, derivative operators [21]. The operator $I_{\lambda}:=I_{1}(r, \lambda)$ was studied recently by Cho and Srivastava [4] and Kim [5].

The operator $I_{r}:=I_{1}(r, 1)$ was studied by Uralgaddi and Somanatha [23]. By using Hadamard product

$$
I_{p}(r, \lambda) f(z):=\mathcal{F}_{\lambda}^{r}(z) * f(z) \quad \text { where } \quad \mathcal{F}_{\lambda}^{r}(z)=z^{p}+\sum_{k=p+1}^{\infty}\left(\frac{k+\lambda}{p+\lambda}\right)^{r} z^{k} \quad(\lambda \geq 0) .
$$

Corresponding to the function $\mathcal{F}_{\lambda}^{r}(z)$ defined by (1.15), we introduce a function $\mathcal{F}_{\lambda, \mu}^{r}(z)$ given by

$$
\mathcal{F}_{\lambda}^{r}(z) * \mathcal{F}_{\lambda, \mu}^{r}(z)=\frac{z^{p}}{(1-z)^{\mu+p-1}}, \quad z \in \mathbb{U}, \mu>0 .
$$

Using $I_{p}(r, \lambda)$, we define the multiplier transformations $T_{\mu}(r, \lambda)$ as follows:

$$
T_{\mu}(r, \lambda) f(z)=\mathcal{F}_{\lambda, \mu}^{r}(z) * f(z) \quad\left(\lambda \geq 0, \mu>0, z \in \mathbb{U}, f \in \mathcal{A}_{p}\right) .
$$

If $f$ is given by (1.1), then by (1.17), we see that

$$
T_{\mu}(r, \lambda) f(z)=z^{p}+\sum_{k=p+1}^{\infty} \Psi_{k}(r, \lambda) a_{k} z^{k},
$$

where

$$
\Psi_{k}(r, \lambda)=\frac{(\mu+p-1)_{k-p}}{(k-p) !}\left(\frac{p+\lambda}{k+\lambda}\right)^{r} .
$$

For $p=1$ we note that a special case of this operator is the integral operator defined in [15]. 
We can verify from the definition (1.17) that

$$
\begin{aligned}
z\left(T_{\mu}(r+1, \lambda) f(z)\right)^{\prime} & =(p+\lambda) T_{\mu}(r, \lambda) f(z)-\lambda T_{\mu}(r+1, \lambda) f(z) \\
z\left(T_{\mu}(r, \lambda) f(z)\right)^{\prime} & =(1-\mu) T_{\mu}(r, \lambda) f(z)+(\mu+p-1) T_{\mu+1}(r, \lambda) f(z) .
\end{aligned}
$$

By using the operator $T_{\mu}(r, \lambda)$ we introduce the following classes of analytic functions for $\phi, \psi \in \mathcal{N}$, and $0 \leq \xi<p, \beta<1$ :

$$
\begin{aligned}
\chi_{\mu}^{*}(r, \lambda ; \xi ; \phi) & :=\left\{f \in \mathcal{A}_{p}: T_{\mu}(r, \lambda) f(z) \in \chi^{*}(\xi ; \phi)\right\} \\
\mathcal{K}_{\mu}^{*}(r, \lambda ; \xi ; \phi) & :=\left\{f \in \mathcal{A}_{p}: T_{\mu}(r, \lambda) f(z) \in \mathcal{K}(\xi ; \phi)\right\} \\
\Phi_{\mu}^{*}(r, \lambda ; \xi ; \beta ; \phi, \psi) & :=\left\{f \in \mathcal{A}_{p}: T_{\mu}(r, \lambda) f(z) \in \Phi(\xi, \beta ; \phi, \psi)\right\} .
\end{aligned}
$$

Also

$$
f(z) \in \mathcal{K}_{\mu}^{*}(r, \lambda ; \xi ; \phi) \Leftrightarrow \frac{z f^{\prime}(z)}{p} \in \chi_{\mu}^{*}(r, \lambda ; \xi, \phi) .
$$

In this paper, basic properties of the classes $\chi_{\mu}(l, m ; \xi, \phi), \mathcal{K}_{\mu}(l, m ; \xi, \phi)$, $\Phi_{\mu}(l, m ; \xi, \beta ; \phi, \psi), \chi_{\mu}^{*}(r, \lambda, \xi, \phi), \mathcal{K}_{\mu}^{*}(r, \lambda, \xi, \phi)$ and $\Phi_{\mu}^{*}(r, \lambda, \xi, \beta, \phi, \psi)$ are studied, such as, inclusion relations and coefficient bounds. Various known or new special cases of our results are also pointed out.

\section{Inclusion properties involving the operator $J_{\mu}^{l, m}$ and $T_{\mu}$}

The following results will be required in our investigation.

Lemma 2.1. ([13]). Let $\phi$ be convex univalent in $\mathbb{U}$ with $\phi(0)=1$ and $\operatorname{Re}[k \phi(z)+\gamma]>0$ $(k, \gamma \in \mathbb{C})$. If $p$ is analytic in $\mathbb{U}$ with $p(0)=1$, then

$$
p(z)+\frac{z p^{\prime}(z)}{k p(z)+\gamma} \prec \phi(z) \quad(z \in \mathbb{U})
$$

implies

$$
p(z) \prec \phi(z), \quad(z \in \mathbb{U}) .
$$

Lemma 2.2. ([13]). Let $\phi$ be convex univalent in $\mathbb{U}$ and let $w$ be analytic in $\mathbb{U}$ with $\operatorname{Re}\{w(z)\} \geq 0$. If $p$ is analytic in $\mathbb{U}$ and $p(0)=\phi(0)$, then

$$
p(z)+w(z) z p^{\prime}(z) \prec \phi(z) \quad(z \in \mathbb{U})
$$

implies

$$
p(z) \prec \phi(z) \quad(z \in \mathbb{U}) .
$$

Theorem 2.3. Let $\mu>1$ and $\phi \in \mathcal{N}$. Then

(i) $\quad \chi_{\alpha_{1}, \mu+1}(l, m ; \xi ; \phi) \subset \chi_{\alpha_{1}, \mu}(l, m ; \xi ; \phi) \subset \chi_{\alpha_{1}+1, \mu}(l, m ; \xi ; \phi) \quad\left(\alpha_{1}>p\right)$

(ii) $\chi_{\mu+1}^{*}(r, \lambda ; \xi ; \phi) \subset \chi_{\mu}^{*}(r, \lambda ; \xi ; \phi) \subset \chi_{\mu}^{*}(r+1, \lambda ; \xi ; \phi)$. 
Proof. (i) First of all, we will show that

$$
\chi_{\alpha_{1}, \mu+1}(l, m ; \xi ; \phi) \subset \chi_{\alpha_{1}, \mu}(l, m ; \xi ; \phi) .
$$

Let $f \in \chi_{\alpha_{1}, \mu+1}(l, m ; \xi ; \phi)$ and set

$$
p(z)=\frac{1}{p-\xi}\left(\frac{z\left(J_{\mu}^{l, m}\left(\alpha_{1}\right) f(z)\right)^{\prime}}{J_{\mu}^{l, m}\left(\alpha_{1}\right) f(z)}-\xi\right),
$$

where $p$ is analytic in $\mathbb{U}$ with $p(0)=1$. Using (1.11) and (2.8),

$$
\frac{1}{p-\xi}\left(\frac{z\left(J_{\mu+1}^{l, m}\left(\alpha_{1}\right) f(z)\right)^{\prime}}{J_{\mu+1}^{l, m}\left(\alpha_{1}\right) f(z)}-\xi\right)=p(z)+\frac{z p^{\prime}(z)}{(p-\xi) p(z)+\mu-1+\xi}, \quad(z \in \mathbb{U})
$$

Since $\mu>1$ and $\phi \in \mathcal{N}$, we see that

$$
\operatorname{Re}\{(p-\xi) \phi(z)+\mu-1+\xi\}>0 \quad\{z \in \mathbb{U}\} .
$$

Using Lemma 2.1 to $(2.9)$, we find that $p \prec \phi$, which means $f \in \chi_{\alpha_{1}, \mu}(l, m ; \xi ; \phi)$.

To prove the second part, let $f \in \chi_{\alpha_{1}, \mu}(l, m ; \xi ; \phi)$ and put

$$
s(z)=\frac{1}{p-\xi}\left(\frac{z\left(J_{\mu}^{l, m}\left(\alpha_{1}+1\right) f(z)\right)^{\prime}}{J_{\mu}^{l, m}\left(\alpha_{1}+1\right) f(z)}-\xi\right),
$$

where $s$ is analytic function with $s(0)=1$. Then, by using the arguments above with (1.10), it follows that $s \prec \phi$ in $\mathbb{U}$, which implies that $f \in \chi_{\alpha_{1}+1, \mu}(l, m ; \xi, \phi)$. Therefore, we complete the proof.

(ii) The proof is the same as (i).

Theorem 2.4. Let $\mu>1$ and $\phi \in \mathcal{N}$. Then,

(i) $\mathcal{K}_{\alpha_{1}, \mu+1}(l, m ; \xi ; \phi) \subset \mathcal{K}_{\alpha_{1}, \mu}(l, m ; \xi ; \phi) \subset \mathcal{K}_{\alpha_{1}+1, \mu}(l, m ; \xi ; \phi), \quad \alpha_{1}>p$

(ii) $\mathcal{K}_{\mu+1}^{*}(r, \lambda ; \xi ; \phi) \subset \mathcal{K}_{\mu}^{*}(r, \lambda ; \xi ; \phi) \subset \mathcal{K}_{\mu}^{*}(r+1, \lambda ; \xi ; \phi)$. 
Proof. Applying (1.13) and Theorem 2.3, we find that

$$
\begin{aligned}
f(z) \in \mathcal{K}_{\alpha_{1}, \mu+1}(l, m ; \xi, \phi) & \Leftrightarrow J_{\mu+1}^{l, m}\left(\alpha_{1}\right) f(z) \in \mathcal{K}(\xi ; \phi) \\
& \Leftrightarrow J_{\mu+1}^{l, m}\left(\alpha_{1}\right) \frac{\left(z f^{\prime}(z)\right)}{p} \in \chi^{*}(\xi ; \phi) \\
& \Leftrightarrow \frac{z f^{\prime}(z)}{p} \in \chi_{\alpha_{1}, \mu+1}(l, m ; \xi ; \phi) \\
& \Rightarrow \frac{z f^{\prime}(z)}{p} \in \chi_{\alpha_{1}, \mu}(l, m ; \xi ; \phi) \\
& \Leftrightarrow \frac{z\left(J_{\mu}^{l, m}\left(\alpha_{1}\right) f(z)\right)^{\prime}}{p} \in \chi^{*}(\xi ; \phi) \\
& \Leftrightarrow J_{\mu}^{l, m}\left(\alpha_{1}\right) f(z) \in \mathcal{K}(\xi ; \phi) \\
& \Leftrightarrow f(z) \in \mathcal{K}_{\alpha_{1}, \mu}(l, m ; \xi ; \phi) \\
f(z) \in \mathcal{K}_{\alpha_{1}, \mu}(l, m ; \xi ; \phi) & \Leftrightarrow \frac{z f^{\prime}(z)}{p} \in \chi_{\alpha_{1}, \mu}(l, m ; \xi ; \phi) \\
& \Rightarrow \frac{z f^{\prime}(z)}{p} \in \chi_{\alpha_{1}+1, \mu}(l, m ; \xi ; \phi) \\
& \Leftrightarrow f(z) \in \mathcal{K}_{\alpha_{1}+1, \mu}(l, m ; \xi ; \phi),
\end{aligned}
$$

which completes the proof.

Now, by using Lemma 2.2, we obtain the following inclusion relation for the class $\Phi_{\alpha_{1}, \mu}(l, m ; \xi, \beta ; \phi, \psi)$ and $\Phi_{\mu}^{*}(l, m ; \xi, \beta ; \phi, \psi)$.

Theorem 2.5. Let $\mu>1$ and $\phi, \psi \in \mathcal{N}$. Then

(i) $\Phi_{\alpha_{1}, \mu+1}(l, m ; \xi, \beta ; \phi, \psi) \subset \Phi_{\alpha_{1}, \mu}(l, m ; \xi, \beta ; \phi, \psi) \subset \Phi_{\alpha_{1}+1, \mu}(l, m ; \xi, \beta ; \phi, \psi), \quad\left(\alpha_{1}>p\right)$

(ii) $\Phi_{\mu+1}^{*}(r, \lambda ; \xi, \beta ; \phi, \psi) \subset \Phi_{\mu}^{*}(r, \lambda ; \xi, \beta ; \phi, \psi) \subset \Phi_{\mu}^{*}(r+1, \lambda ; \xi, \beta ; \phi, \psi)$.

Proof. First, we will prove that

$$
\Phi_{\alpha_{1}, \mu+1}(l, m ; \xi, \beta ; \phi, \psi) \subset \Phi_{\alpha_{1}, \mu}(l, m ; \xi, \beta ; \phi, \psi) .
$$

Let $f(z) \in \Phi_{\alpha_{1}, \mu+1}(l, m ; \xi, \beta ; \phi, \psi)$. Then, from the definition of $\Phi_{\alpha_{1}, \mu+1}(l, m ; \xi, \beta ; \phi, \psi)$, there exists a function $r(z) \in \chi^{*}(\xi, \phi)$ such that

$$
\frac{1}{p-\beta}\left(\frac{z\left(J_{\mu+1}^{l, m}\left(\alpha_{1}\right) f(z)\right)^{\prime}}{r(z)}-\beta\right) \prec \psi(z) \quad(z \in \mathbb{U}) .
$$

Choose the function $g(z)$ such that $J_{\mu+1}^{l, m}\left(\alpha_{1}\right) g(z)=r(z)$. Then, $g(z) \in \chi_{\alpha_{1}, \mu+1}(l, m ; \xi ; \phi)$ and

$$
\frac{1}{p-\beta}\left(\frac{z\left(J_{\mu+1}^{l, m}\left(\alpha_{1}\right) f(z)\right)^{\prime}}{J_{\mu+1}\left(\alpha_{1}\right) g(z)}-\beta\right) \prec \psi(z) \quad(z \in \mathbb{U}) .
$$


Now let

$$
p(z)=\frac{1}{p-\beta}\left(\frac{z\left(J_{\mu}^{l, m}\left(\alpha_{1}\right) f(z)\right)^{\prime}}{J_{\mu}^{l, m}\left(\alpha_{1}\right) g(z)}-\beta\right),
$$

where $p$ is analytic in $\mathbb{U}$ with $p(0)=1$. Using (1.11), we have

$$
\begin{aligned}
(p-\beta) z p^{\prime}(z) J_{\mu}^{l, m}\left(\alpha_{1}\right) g(z)+((p-\beta) p(z)+\beta) z\left(J_{\mu}^{l, m}\left(\alpha_{1}\right) g(z)\right)^{\prime} \\
=(\mu+p-1) z\left(J_{\mu+1}^{l, m}\left(\alpha_{1}\right) f(z)\right)^{\prime}-(\mu-1) z\left(J_{\mu}^{l, m}\left(\alpha_{1}\right) f(z)\right)^{\prime} .
\end{aligned}
$$

Since $g(z) \in \chi_{\alpha_{1}, \mu+1}(l, m ; \xi ; \phi)$, by Theorem 2.3, we know that $g(z) \in \chi_{\alpha_{1}, \mu}(l, m ; \xi ; \phi)$. Let

$$
q(z)=\frac{1}{p-\xi}\left(\frac{z\left(J_{\mu}^{l, m}\left(\alpha_{1}\right) g(z)\right)^{\prime}}{J_{\mu}^{l, m}\left(\alpha_{1}\right) g(z)}-\xi\right) .
$$

Then, using (1.11), once again, we have

$$
(\mu+p-1) \frac{J_{\mu+1}^{l, m}\left(\alpha_{1}\right) g(z)}{J_{\mu}\left(\alpha_{1}\right) g(z)}=(p-\xi) q(z)+\mu-1+\xi .
$$

From $(2.21),(2.23)$, we obtain

$$
\frac{1}{p-\beta}\left(\frac{z\left(J_{\mu+1}^{l, m}\left(\alpha_{1}\right) f(z)\right)^{\prime}}{J_{\mu+1}^{l, m}\left(\alpha_{1}\right) g(z)}-\beta\right)=p(z)+\frac{\left.z p^{\prime} z\right)}{(p-\xi) q(z)+\mu-1+\xi} .
$$

Since $\mu>1$ and $q \prec \phi$ in $\mathbb{U}$,

$$
\operatorname{Re}\{(p-\xi) q(z)+\mu-1+\xi\}>0 \quad(z \in \mathbb{U}) .
$$

Therefore, from Lemma 2.2 , we can show that $p \prec \psi$, so that $f \in \Phi_{\alpha_{1}, \mu}(l, m ; \xi, \beta ; \phi, \psi)$.

For the second part, by using the same arguments above with (1.10), we obtain

$$
\Phi_{\alpha_{1}, \mu}(l, m ; \xi, \beta ; \phi, \psi) \subset \Phi_{\alpha_{1}+1, \mu}(l, m ; \xi, \beta ; \phi, \psi) .
$$

Therefore, we complete the proof.

\section{Inclusion Properties Involving the Integral Operator $F_{a}$}

In this section, we consider the generalized Libera integral operator $F_{a}[17]$ defined by

$$
F_{a}(f):=F_{a}(f)(z)=\frac{a+1}{z^{a}} \int_{0}^{z} t^{a-1} f(t) d t \quad\left(f \in \mathcal{A}_{p}, a>-1\right) .
$$

First we will prove the following.

Theorem 3.1.

(i) If $f \in \chi_{\alpha_{1}, \mu}(l, m ; \xi ; \phi)$, then $F_{a}(f) \in \chi_{\alpha_{1}, \mu}(l, m ; \xi ; \phi)(a \geq 0)$.

(ii) If $f \in \chi_{\mu}^{*}(r, \lambda ; \xi ; \phi)$, then $F_{a}(f) \in \chi_{\mu}^{*}(r, \lambda ; \xi ; \phi)(a \geq 0)$. 
Proof. (i) Let $f \in \chi_{\alpha_{1}, \mu}(l, m ; \xi ; \phi)$ and set

$$
p(z)=\frac{1}{p-\xi}\left(\frac{z\left(J_{\mu}^{l, m}\left(\alpha_{1}\right) F_{a}(f)(z)\right)^{\prime}}{J_{\mu}^{l, m}\left(\alpha_{1}\right) F_{a}(f)(z)}-\xi\right)
$$

where $p$ is analytic in $\mathbb{U}$ with $p(0)=1$. From (3.1), we have

$$
z\left(J_{\mu}^{l, m}\left(\alpha_{1}\right) F_{a}(f)(z)\right)^{\prime}=(a+1) J_{\mu}^{l, m}\left(\alpha_{1}\right) f(z)-a J_{\mu}^{l, m}\left(\alpha_{1}\right) F_{a}(f)(z) .
$$

Then, by using (3.2) and (3.3), we obtain

$$
(a+1) \frac{J_{\mu}^{l, m}\left(\alpha_{1}\right) f(z)}{J_{\mu}^{l, m}\left(\alpha_{1}\right) F_{a}(f)(z)}=(p-\xi) p(z)+a+\xi .
$$

Taking the logarithmic differentiation on both sides of (3.4) and multiplying by $z$, we have

$$
p(z)+\frac{z p^{\prime}(z)}{(p-\xi) p(z)+a+\xi}=\frac{1}{p-\xi}\left(\frac{z\left(J_{\mu}^{l, m}\left(\alpha_{1}\right) f(z)\right)^{\prime}}{J_{\mu}^{l, m}\left(\alpha_{1}\right) f(z)}-\xi\right) \quad(z \in \mathbb{U}) .
$$

Hence, by virtue of Lemma 2.1, we conclude that $p \prec \phi$ in $\mathbb{U}$, which implies that $F_{a}(f) \in$ $\chi_{\alpha_{1}, \mu}(l, m ; \xi ; \phi)$.

(ii) The proof is the same as (i).

Next, we derive an inclusion property involving $F_{a}$, which is given by the following.

\section{Theorem 3.2.}

(i) If $f \in \mathcal{K}_{\alpha_{1}, \mu}(l, m ; \xi ; \phi)$, then $F_{a}(f) \in \mathcal{K}_{\alpha_{1}, \mu}(l, m ; \xi ; \phi) \quad(a \geq 0)$.

(ii) If $f \in \mathcal{K}_{\mu}^{*}(r, \lambda ; \xi ; \phi)$, then $F_{a}(f) \in \mathcal{K}_{\mu}^{*}(r, \lambda ; \xi ; \phi) \quad(a \geq 0)$.

Proof. By applying Theorem 3.1, it follows that

$$
\begin{aligned}
f(z) \in \mathcal{K}_{\alpha_{1}, \mu}(l, m ; \xi ; \phi) & \Leftrightarrow \frac{z f^{\prime}(z)}{p} \in \chi_{\alpha_{1}, \mu}(l, m ; \xi ; \phi) \\
& \Rightarrow F_{a}\left(\frac{z f^{\prime}(z)}{p}\right) \in \chi_{\alpha_{1}, \mu}(l, m ; \xi ; \phi) \\
& \Leftrightarrow z \frac{\left(F_{a}(f)(z)\right)^{\prime}}{p} \in \chi_{\alpha_{1}, \mu}(l, m ; \xi ; \phi) \\
& \Leftrightarrow F_{a}(f)(z) \in \mathcal{K}_{\alpha_{1}, \mu}(l, m ; \xi ; \phi) .
\end{aligned}
$$

From Theorems 3.1 and 3.2, we have the following.

\section{Theorem 3.3.}

(i) If $f \in \Phi_{\alpha_{1}, \mu}(l, m ; \xi, \beta ; \phi, \psi)$, then $F_{a}(f) \in \Phi_{\alpha_{1}, \mu}(l, m ; \xi, \beta ; \phi, \psi), \quad(a \geq 0)$.

(ii) If $f \in \Phi_{\mu}^{*}(r, \lambda ; \xi, \beta ; \phi, \psi)$, then $F_{a}(f) \in \Phi_{\mu}^{*}(r, \lambda ; \xi, \beta ; \phi, \psi), \quad(a \geq 0)$. 
Proof. Let $f \in \Phi_{\alpha_{1}, \mu}(l, m ; \xi, \beta ; \phi, \psi)$. Then, from the definition of the class $\Phi_{\alpha_{1}, \mu}(l, m ; \xi$, $\beta ; \phi, \psi)$, there exists a function $g(z) \in \chi_{\alpha_{1}, \mu}(l, m ; \xi ; \phi)$ such that

$$
\frac{1}{p-\beta}\left(\frac{z\left(J_{\mu}^{l, m}\left(\alpha_{1}\right) f(z)\right)^{\prime}}{J_{\mu}^{l, m}\left(\alpha_{1}\right) g(z)}-\beta\right) \prec \psi(z) \quad(z \in \mathbb{U}) .
$$

Thus, we set

$$
p(z)=\frac{1}{p-\beta}\left(\frac{z\left(J_{\mu}^{l, m}\left(\alpha_{1}\right) F_{a}(f)(z)\right)^{\prime}}{J_{\mu}^{l, m}\left(\alpha_{1}\right) F_{a}(g)(z)}-\beta\right),
$$

where $p$ is analytic in $\mathbb{U}$ with $p(0)=1$. Since $g(z) \in \chi_{\alpha_{1}, \mu}(l, m ; \xi ; \phi)$, we see from Theorem 3.1 that $F_{a}(g) \in \chi_{\alpha_{1}, \mu}(l, m ; \xi ; \phi)$. Using (3.3), we have

$$
((p-\beta) p(z)+\beta) J_{\mu}^{l, m}\left(\alpha_{1}\right) F_{a}(g)(z)+a J_{\mu}^{l, m}\left(\alpha_{1}\right) F_{a}(f)(z)=(a+1) J_{\mu}^{l, m}\left(\alpha_{1}\right) f(z) .
$$

By some calculation, we get

$$
(a+1) \frac{z\left(J_{\alpha}^{l, m}\left(\alpha_{1}\right) f(z)\right)^{\prime}}{J_{\mu}^{l, m}\left(\alpha_{1}\right) F_{a}(g)(z)}=((p-\beta) p(z)+\beta)((p-\xi) q(z)+a+\xi)+(p-\beta) z p^{\prime}(z),
$$

where

$$
q(z)=\frac{1}{p-\xi}\left(\frac{z\left(J_{\mu}^{l, m}\left(\alpha_{1}\right) F_{a}(g)(z)\right)^{\prime}}{J_{\mu}^{l, m}\left(\alpha_{1}\right) F_{a}(g)(z)}-\xi\right) .
$$

Hence, we have

$$
\frac{1}{p-\beta}\left(\frac{z\left(J_{\mu}^{l, m}\left(\alpha_{1}\right) f(z)\right)^{\prime}}{J_{\mu}^{l, m}\left(\alpha_{1}\right) g(z)}-\beta\right)=p(z)+\frac{z p^{\prime}(z)}{(p-\xi) q(z)+a+\xi} .
$$

The remaining part of the proof in Theorem 3.3 is similar to that of Theorem 2.5 and so we leave it.

\section{Coefficient bounds}

Now we will give bounds for the coefficients of series expansion of functions belonging to the classes $\chi_{\alpha_{1}, \mu}(\xi), \mathcal{K}_{\alpha_{1}, \mu}(\xi), \chi_{\mu}^{*}(\xi)$ and $\mathcal{K}_{\mu}^{*}(\xi)$.

Taking into account the fundamental relation

$$
\phi(z)=\frac{1}{p-\xi}\left(\frac{z\left(J_{\mu}^{l, m}\left(\alpha_{1}\right) f_{\xi}(z)\right)^{\prime}}{J_{\mu}^{l, m}\left(\alpha_{1}\right) f_{\xi}(z)}-\xi\right),
$$

between the extremal functions in the class $P(\phi(z))$ and the extremal functions $f_{\xi}$ of the class $\chi_{\alpha_{1}, \mu}(\xi)$ and in view of (1.9) and (4.1), we have for $\phi(z)=1+\sum_{k=1}^{\infty} B_{k} z^{k}$,

$$
f_{\xi}=z^{p}+\sum_{k-p+1}^{\infty} A_{k} z^{k}
$$


a coefficient relation

$$
(k-p) A_{k} \Psi_{k}\left(\alpha_{1}, \mu\right)=(p-\xi) \sum_{j=p}^{k-1} B_{k-j} A_{j} \Psi_{j}\left(\alpha_{1}, \mu\right), \quad A_{p}=1 .
$$

In particular, by a straightforward computation, we obtain

$$
A_{p+1}=\frac{(p-\xi) B_{1}}{\Psi_{p+1}\left(\alpha_{1}, \mu\right)} .
$$

Observe that the coefficients $A_{k}$ are nonnegative since $\Psi_{k}\left(\alpha_{1}, \mu\right) \geq 0$.

We give sharp bound on the second coefficient for functions of the classes, $\chi_{\alpha_{1}, \mu}(\xi)$ and $\chi_{\mu}^{*}(\xi)$.

Theorem 4.1. i) If a function of the form (1.1) is in $\chi_{\alpha_{1}, \mu}(\xi)$, then

$$
\left|a_{k}\right| \leq \frac{(p-\xi)}{\Psi_{k}\left(\alpha_{1}, \mu\right)} \frac{\left(\left|B_{1}\right|\right)_{k-p}}{(1)_{k-p}}, \quad k \geq p+1 .
$$

(ii) If a function of the form (1.1) is in $\chi_{\mu_{1}}^{*}(\xi)$, then

$$
\left|a_{k}\right| \leq \frac{(p-\xi)}{\Psi_{k}(r, \mu)} \frac{\left(\left|B_{1}\right|\right)_{k-p}}{(1)_{k-p}}, \quad k \geq p+1
$$

For the proof of this theorem, we need the following result by Rogosinski [20].

Rogosinski's Theorem. Let $h(z)=1+\sum_{k=1}^{\infty} c_{k} z^{k}$ be subordinate to $H(z)=1+\sum_{k=1}^{\infty} C_{k} z^{k}$ in $\mathbb{U}$. If $H(z)$ is univalent in $\mathbb{U}$ and $H(\mathbb{U})$ is convex, then $\left|c_{k}\right| \leq\left|C_{1}\right|, \quad k \geq 1$.

Proof of Theorem 4.1. (i) Let $f \in \chi_{\alpha_{1}, \mu}(\xi), f(z)=z^{p}+\sum_{k=p+1}^{\infty} a_{k} z^{k}$, we obtain

$$
\frac{1}{p-\xi}\left(\frac{z\left(J_{\mu}^{l, m}\left(\alpha_{1}\right) f(z)\right)^{\prime}}{J_{\mu}^{l, m}\left(\alpha_{1}\right) f(z)}-\xi\right) \prec \phi(z) .
$$

Define $q(z)=\frac{1}{p-\xi}\left(\frac{z\left(J_{\mu}^{l, m}\left(\alpha_{1}\right) f(z)\right)^{\prime}}{J_{\mu}^{l, m}\left(\alpha_{1}\right) f(z)}-\xi\right)=1+\sum_{k=1}^{\infty} c_{k} z^{k}$. The function $\phi$ is univalent in $\mathbb{U}$ and $\phi(\mathbb{U})$, the conic domain, is convex domain, so Rogosinski's theorem applies. Then we have

$$
\left|c_{k}\right| \leq 2, \quad k \geq 1 .
$$

Now writing $((p-\xi) q(z)+\xi) J_{\mu}^{l, m}\left(\alpha_{1}\right) f(z)=z\left(J_{\mu}^{l, m}\left(\alpha_{1}\right) f(z)\right)^{\prime}$ and comparing coefficients of $z^{k}$ on both sides, we get

$$
(k-p) a_{k} \Psi_{k}\left(\alpha_{1}, \mu\right)=(p-\xi) \sum_{j=p}^{k-1} c_{k-j} a_{j} \Psi_{j}\left(\alpha_{1}, \mu\right), \quad a_{p}=1 .
$$


From (4.6), we get $\left|a_{p+1}\right|=\frac{(p-\xi)}{\Psi_{p+1}\left(\alpha_{1}, \mu\right)}\left|c_{1}\right| \leq \frac{(p-\xi)\left|B_{1}\right|}{\Psi_{p+1}\left(\alpha_{1}, \mu\right)}$. So the result is true for $k=p+1$. Let $k \geq p+1$ and assume that the inequality (4.4) is true for all $j \leq k-1$. By using (4.5), (4.6) and applying the induction hypothesis to $\left|a_{j}\right|$, we get

$$
\begin{aligned}
\left|a_{k}\right| & \leq \frac{(p-\xi)}{(k-p) \Psi_{k}\left(\alpha_{1}, \mu\right)}\left[\left|c_{1}\right|+\sum_{j=p+1}^{k-1}\left|c_{k-j}\right|\left|a_{j}\right| \Psi_{j}\left(\alpha_{1}, \mu\right)\right] \\
& \leq \frac{(p-\xi)\left|B_{1}\right|}{(k-p) \Psi_{k}\left(\alpha_{1}, \mu\right)}\left[1+\sum_{j=p+1}^{k-1} \frac{(p-\xi)\left(\left|B_{1}\right|\right)_{j-p}}{(1)_{j-p}}\right] .
\end{aligned}
$$

Putting $p-1 \leq \xi<p$, we obtain

$$
\left|a_{k}\right| \leq \frac{(p-\xi)\left|B_{1}\right|}{(k-p) \Psi_{k}\left(\alpha_{1}, \mu\right)}\left[1+\sum_{j=p+1}^{k-1} \frac{\left(\left|B_{1}\right|\right)_{j-p}}{(1)_{j-p}}\right] .
$$

By applying mathematical induction another time, we find that

$$
1+\sum_{j=p+1}^{k-1} \frac{\left.\left|B_{1}\right|\right)_{j-p}}{(1)_{j-p}}=\frac{\left(\left|B_{1}\right|+1\right)\left(\left|B_{1}\right|+2\right) \cdots\left(\left|B_{1}\right|+k-p-1\right)}{(k-p-1) !} .
$$

Thus we get the inequality (4.4).

(ii) The proof is the same as (i).

Applying the relation (1.13) and (1.22), we observe that the extremal function $\mathcal{K}_{\alpha_{1}, \mu}(\xi)$ and $\mathcal{K}_{\mu}^{*}(\xi)$ denoted by $F_{\xi}(z)$, is given by

$$
F_{\xi}(z)=p \int_{0}^{z} \frac{f_{\xi}(\gamma)}{\gamma} d \gamma
$$

where $f_{\xi}(z)$ is given by (4.2).

By (4.3) and for

$$
F_{\xi}(z)=z+\sum_{k=p+1}^{\infty} C_{k} z^{k}
$$

we get

$$
C_{p+1}=\frac{p(p-\xi) B_{1}}{(p+1) \Psi_{p+1}\left(\alpha_{1}, \mu\right)} .
$$

Applying relation (1.13), we can prove the next result.

\section{Corollary 4.2.}

(i) If a function $f$ of the form (1.1) is in $\mathcal{K}_{\alpha_{1}, \mu}(\xi)$, then

$$
\left|a_{k}\right| \leq \frac{p(p-\xi)\left(\left|B_{1}\right|\right)_{k-p}}{k \Psi_{k}\left(\alpha_{1}, \mu\right)(1)_{k-p}} .
$$


(ii) If a function $f$ of the form (1.1) is in $\mathcal{K}_{\mu}^{*}(\xi)$, then

$$
\left|a_{k}\right| \leq \frac{p(p-\xi)\left(\left|B_{1}\right|\right)_{k-p}}{k \Psi_{k}(r, \mu)(1)_{k-p}}
$$

\section{References}

[1] H. A. Al-Kharsani and R. A. Al-Khal, Generalized integral operator and univalent functions, Int. J. Math. Anal. 1 (2007), 709-718.

[2] S.D. Bernardi, Convex and starlike univalent functions, Trans. Amer. Math. Soc. 135(1969), 429-446.

[3] B. C. Carlson and D. B. Shaffer, Starlike and prestarlike hypergeometric functions, SIAM J. Math. Anal. 15 (1984), 737-745.

[4] N. E. Cho and H. M. Srivastava, Argument estimates of certain analytic functions defined by a class of multiplier transformations, Math. Comput. Modeling 37 (2003), 39-43.

[5] N.E. Cho and T. H. Kim, Multiplier transformations and strongly close-to-convex functions, Bull. Korean Math. Soc. 40 (2003), 399-410.

[6] J. Dziok and H.M. Srivastava, Certain subclasses of analytic functions associated with the generalized hypergeometric function, Integral Transforms Spec. Funct. 14 (2003), 7-18.

[7] J. Dziok and H.M. Srivastava, Classes of analytic functions associated with the generalized hypergeometric function. Appl. Math. Comput. 103 (1999), 1-13.

[8] P. Eenigenburg, S.S. Miller, P.T. Mocanu, and M.O. Reade, On a Briot-Bouqut differential subordination, Int. Sch. Num. Math. 28 (19 83), 157-172.

[9] Ju. E. Hohlov, Operators and operations on the class of univalent functions, Izv. Vyssh. Uchebn. Zaved. Math. 1978, 10 (1978), 83-89.

[10] O. S. Kwon and N.E. Cho, Inclusion properties for certain subclasses of analytic functions associated with the Dziok-Srivastava operator, J. Ineq. and Appl., Vol. (2007), 10 pages.

[11] R. J. Libera, Some classes of regular univalent functions, Proc. Amer. Math. Soc. 16(1965), $755-758$.

[12] A. E. Livingston, On the radius of univalence of certain analytic functions, Proc. Amer. Math. Soc. 17(1966), 352-357.

[13] S. S. Miller and P. T. Mocanu, Differential Subordinations and univalent functions, The Michigan Mathematical Journal, 28 (1981), 157-172.

[14] K. I. Noor and M. A. Noor, On integral operators, J. Math. Anal. and Appl., 23 (1999), 341-352.

[15] K. I. Noor, On some analytic functions defined by a multiplier transformation, Int. J. Math. \& Math. Scie., (2007), 9 pages.

[16] S. Owa, On the distoriton theorems I, Kyungpook Math. J., 18 (1978), 53-59.

[17] S. Owa and H.M. Srivastava, Some applications of the generalized Libera integral operarator, Proceedings of the Japan Academy, Series A, (62) (1986), 125-128.

[18] S. Owa and H.M. Srivastava, Univalent and starlike generalized hypergeometric functions, Canad. J. Math., 39 (1987), 1057-1077.

[19] St. Ruscheweyh, New criteria for univalent functions, Proc. Amer. Math. Soc., 49(1975), 109-115. 
[20] W. Rogosinski, On the coefficients of subordinate functions, Proc. London Math. Soc. 48(1943), 48-82.

[21] G.S. Şălăgean, Subclasses of univalent functions in Complex analysis, Springer, Berlin 1(1981), 362-372.

[22] H.M. Srivastava, Some families of fractional derivative and other linear operators associated with analytic univalent and multivalent functions in Analysis and its applications, Allied Publ., New Delhi, (2000), 209-243.

[23] B.A. Uralegaddi and C. Somanatha, Certain classes of univalent functions in Current topics in analytic function theory, World Publishing, River Edge, NJ, 1992,

Department of Mathematics, Faculty of Science, P.O. Box 838, Dammam 31113, Kingdom of Saudi Arabia.

E-mail: hakh73@hotmail.com

Department of Mathematics, Scott Christian College Nagercoil 629 003, Tamil Nadu, India.

E-mail: najarifi@hotmail.com 